\title{
Modeling and Dynamic Analysis of Telescopic Systems of Structural Members with Clearance
}

\author{
P. BARTHELS and J. WAUER \\ Institut für Technische Mechanik, Universität Karlsruhe, D-76128 Karlsruhe, \\ Germany
}

February 18, 2005

\begin{abstract}
The dynamic behavior of multi-section constructions with clearance during extending and retracting motion of the sections is analyzed. First, an appropriate physical modeling is introduced before next, the governing boundary value problem is derived by applying Hamilton's principle. Then, a classical discretization procedure is used to generate a coupled system of nonlinear ordinary differential equations as the corresponding truncated mathematical model. Performing appropriate simulations to be verified by results of an alternative software package and partly validated by some preliminary experiments, the vibrational behavior of the suggested type of multi-section telescoping systems can be studied in detail.
\end{abstract}

Keywords: telescopic systems, multi-section constructions, clearance, vibrational behavior.

\section{Introduction}

Graduated multi-section systems of structural components extending and retracting inside each other are interesting technical systems, e.g., mobile cranes, rack feeder, etc. (see [1], for example and Fig. 1). Due to overall rigid body translation or slew maneuvers combined with the

Figure 1. Rack feeder.

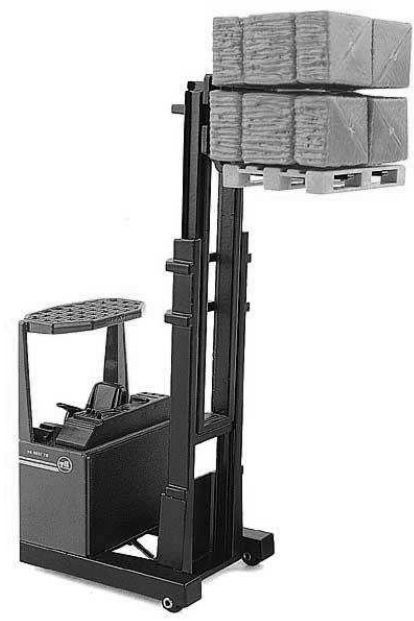

(C) 2005 Kluwer Academic Publishers. Printed in the Netherlands. 
extending and retracting motion of the sections, bending vibrations of the system perpendicular to the telescopic axis occur. In technical applications these vibrations lead to a reduction of the efficiency and to safety problems so that a controlled vibration suppression seems to be useful.

A first step to develop efficient and safe multi-section constructions is an appropriate modeling of such systems and the examination of the vibrational behavior, which is the objective of the present contribution. Then, a controller concept for preventing the harmful vibrations can be developed in a second step, to be presented as another paper in the near future.

Considering the introduced physical model, Hamilton's principle is applied to derive the governing boundary value problem. Galerkin's method based on admissible shape functions is used as a discretization procedure to generate a system of coupled ordinary differential equations. For extended parameter studies of multi-section telescopic systems, a program based on the commercial software package MAPLE is implemented to generate the equations of motion automatically starting with the system parameters. In general, the equations of motion are nonlinear and time-variant. Performing appropriate simulations, the vibrational behavior of multi-section telescoping systems can be studied in detail.

\section{Physical Model}

From the viewpoint of mechanics, a non-linear field problem of vibrating structural members with variable geometry has to be considered. Material surface areas of particular components move along surface areas of other components and define complicated boundary and transition conditions. The clearance produces non-linear effects. In many applications, e.g., the rack feeder shown in Fig. $\mathbf{1}$ or for mobile cranes, the different segments are slender and can be modeled as Bernoulli/Euler beams mounted on a rigid vehicle unit and carrying at some location, e.g., at the end of the last section, a load unit assumed to be rigid. The vehicle unit together with the first deformable segment and all the other segments (one of them together with the load) perform transverse motions and the extending or retracting motion of the sections is supplemented. The vehicle motion is either prescribed owing to ideal sources of energy or controlled by real power supplies, resulting in bending vibrations of the beams. The contact regions between two sections are modeled as discrete point contacts. A special feature of the modeling is to introduce the reaction forces at the contact points in the 
form of distributed line loads (by using Dirac impulse functions), so that for the contacting sections elementary boundary conditions remain. The contact formulation itself takes place via one-sided spring-damper elements.

The procedure is illustrated in Fig. 2a for a two-section telescopic beam system mounted on a rigid traverse performing a translational motion accompanied by an extending motion of the two beam segments with defined clearance between them.

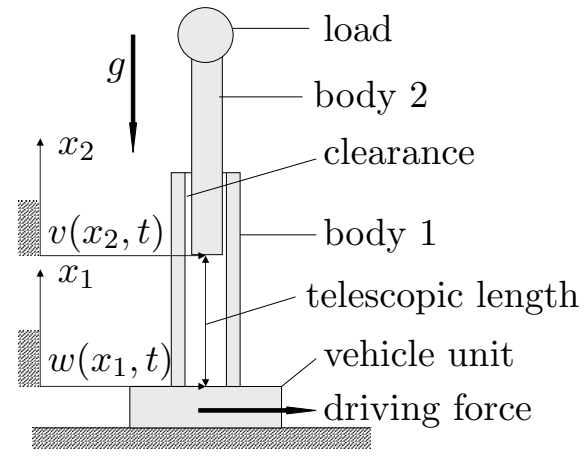

a)

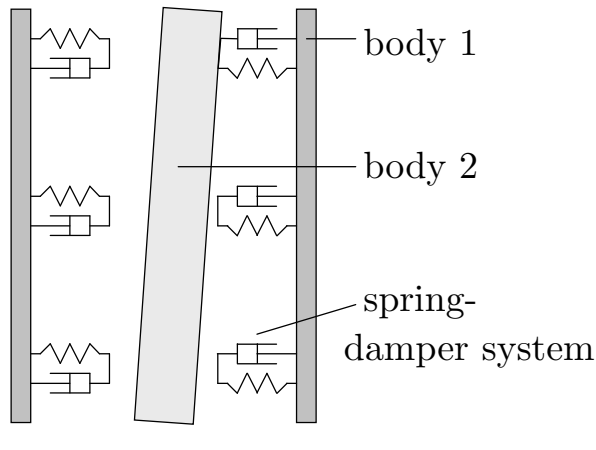

b)

Figure 2. a) System model, b) Contact formulation.

Beam 1 is fixed at a rigid vehicle unit; beam 2 carries a point load at its end. The vehicle is driven by a horizontal force $F$ as excitation of the system. The deformation of the beams (including vehicle mass and load) is represented by the absolute displacements $w\left(x_{1}, t\right)$ and $v\left(x_{2}, t\right)$. The model is defined by the following parameters: beam lengths $l_{1,2}$, cross-sectional areas $A_{1,2}$, cross-sectional moments of inertia $I_{1,2}$, density $\rho$ and Young's modulus $E$ of the two flexible components, masses of load and vehicle $m_{L}$ and $m_{T}$, respectively, and telescopic length $l_{A}(t)$. The contact between the beams is realized (see Fig. 2b) via discrete spring-damper systems in the form of a so-called displacement condition (not a force condition) [2], the given number $n$ of contact points, the clearance $l_{S}$, spring stiffness $c$, and damping coefficient $d$. $c$ can be estimated from the geometry and the material of the contact partners whereas the estimation of $d$ is more complicated. As the purpose of the model is the creation of a control concept for vibration suppression, it is important that the equations of motion stay as simple as possible. In the controlled system the clearance plays the role of an external disturbance and as the controller has to work for every kind of contact, a very accurate estimation of $d$ is not necessary. In the axial direction it is assumed that there is no friction. This assumption is justifiable as the 
bearing between the different segments is realized as roller bearing in many applications. The free body diagram in Fig. 3 shows the reactive forces (contact and axial forces) between the two bodies. It is assumed here that the force flow leads from the upper part into the lower part. It has to be noticed that the model has to be modified (which simplifies the modeling) for specific applications when the telescopic motion of the upper part is induced by an external system so that the lower part is not pre-stressed.

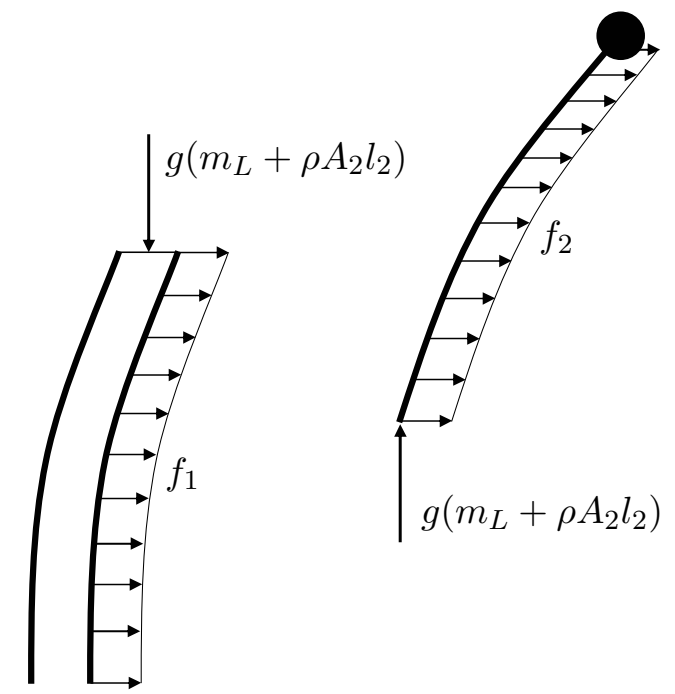

Figure 3. Free body diagram.

\section{Formulation}

\subsection{Boundary VAlue PROBlem}

Applying Hamilton's principle

$$
\delta \int_{t_{0}}^{t_{1}}(T-U) d t+\int_{t_{0}}^{t_{1}} W_{\text {virt }} d t=0
$$

the governing boundary value problem can be derived. $T$ is the kinetic energy, $U$ the potential energy and $W_{\text {virt }}$ the virtual work of forces without potential of the considered system. If beam segments of uniform cross-sectional properties $\rho A_{1}, \rho A_{2}, E I_{1}, E I_{2}$ are assumed, the kinetic energy reads 


$$
T=\frac{1}{2} \int_{0}^{l_{1}} \rho A_{1}^{*} w_{t}^{2} d x_{1}+\frac{1}{2} \int_{0}^{l_{2}} \rho A_{2}^{*} v_{t}^{2} d x_{2}
$$

where $\rho A_{1}^{*}=\rho A_{1}+m_{T} \delta\left(x_{1}\right)$ and $\rho A_{2}^{*}=\rho A_{2}+m_{L} \delta\left(x_{2}-l_{2}\right)$. The symbol $\delta($.$) represents Dirac's delta-function to include the locally$ concentrated masses of vehicle and load into the mass distribution of the corresponding bodies. If the action of the spring-damper systems is completely included into the virtual work, for the remaining potential energy one obtains

$$
\begin{aligned}
U= & \frac{1}{2} \int_{0}^{l_{1}}\left[E I_{1} w_{x_{1} x_{1}}^{2}-\rho g\left(\int_{x_{1}}^{l_{1}} A_{1}^{*} d \bar{x}_{1}+\int_{0}^{l_{2}} A_{2}^{*} d x_{2}\right) w_{x_{1}}^{2}\right] d x_{1} \\
& +\frac{1}{2} \int_{0}^{l_{2}}\left[E I_{2} v_{x_{2} x_{2}}^{2}-\rho g \int_{x_{2}}^{l_{2}} A_{2}^{*} d \bar{x}_{2} v_{x_{2}}^{2}\right] d x_{2} .
\end{aligned}
$$

Since no internal damping of the beam segments will be taken into consideration, as the worst case for control, the virtual work contains all the contact forces between the beams and the locally concentrated driving force of the vehicle as distributed loads $f_{1}\left(x_{1}, t\right)$ and $f_{2}\left(x_{2}, t\right)$ (see Fig. 3) which couple the resulting field equations:

$$
W_{\text {virt }}=\int_{0}^{l_{1}} f_{1} \delta w d x_{1}+\int_{0}^{l_{2}} f_{2} \delta v d x_{2} .
$$

Due to the formulation of all these locally concentrated forces by distributed loads using Dirac impulses, the boundary conditions will be homogeneous. Evaluating Hamilton's principle (1) introducing $T$, $U$ and $W_{\text {virt }}$ according to eqs. (2), (3) and (4), respectively, yields the governing field equations

$$
\begin{aligned}
\rho A_{1}^{*} w_{t t} & +E I_{1} w_{x_{1} x_{1} x_{1} x_{1}}+\rho A_{1} g\left[\left(l_{1}-x_{1}\right) w_{x_{1}}\right]_{x_{1}}+g\left(m_{L}+\rho A_{2} l_{2}\right) w_{x_{1} x_{1}} \\
& =f_{1}\left(x_{1}, t\right)+\delta\left(x_{1}-l_{1}\right) g\left(m_{L}+\rho A_{2} l_{2}\right) w_{x_{1}} \\
\rho A_{2}^{*} v_{t t} & +E I_{2} v_{x_{2} x_{2} x_{2} x_{2}}+\rho A_{2} g\left[\left(l_{2}-x_{2}\right) v_{x_{2}}\right]_{x_{2}}+g m_{L} v_{x_{2} x_{2}} \\
& =f_{2}\left(x_{2}, t\right)-\delta\left(x_{2}\right) g\left(m_{L}+\rho A_{2} l_{2}\right) v_{x_{2}}+\delta\left(x_{2}-l_{2}\right) g m_{L} v_{x_{2}}
\end{aligned}
$$

and the corresponding boundary conditions

$$
\begin{gathered}
w_{x_{1}}(0, t)=0, w_{x_{1} x_{1} x_{1}}(0, t)=0, w_{x_{1} x_{1}}\left(l_{1}, t\right)=0, w_{x_{1} x_{1} x_{1}}\left(l_{1}, t\right)=0 \\
v_{x_{2} x_{2}}(0, t)=0, v_{x_{2} x_{2} x_{2}}(0, t)=0, v_{x_{2} x_{2}}\left(l_{2}, t\right)=0, v_{x_{2} x_{2} x_{2}}\left(l_{2}, t\right)=0
\end{gathered}
$$

for the two bodies. 
For the special case in which the beam segments contact each other at the two points $x_{1}=l_{1}$ and $x_{2}=0$ only, the distributed forces are specified as

$$
\begin{aligned}
f_{1}= & \delta\left(x_{1}\right) F+\delta\left(x_{1}-l_{A}(t)\right)\left(F_{K}\left(\xi_{1}(t)\right)+\frac{d}{d t} \xi_{1}(t) \cdot D_{K}\left(\xi_{1}(t)\right)\right) \\
& +\delta\left(x_{1}-l_{1}\right)\left(F_{K}\left(\xi_{2}(t)\right)+\frac{d}{d t} \xi_{2}(t) \cdot D_{K}\left(\xi_{2}(t)\right)\right) \\
f_{2}= & -\delta\left(x_{2}\right)\left(F_{K}\left(\xi_{1}(t)\right)+\frac{d}{d t} \xi_{1}(t) \cdot D_{K}\left(\xi_{1}(t)\right)\right) \\
& -\delta\left(x_{2}-\left(l_{1}-l_{A}(t)\right)\right)\left(F_{K}\left(\xi_{2}(t)\right)+\frac{d}{d t} \xi_{2}(t) \cdot D_{K}\left(\xi_{2}(t)\right)\right)
\end{aligned}
$$

where

$$
\begin{array}{r}
F_{K}(\xi(t))=c\left[\xi(t)-\frac{1}{2}\left(\xi(t)+\frac{l_{S}}{2}\right) \operatorname{sign}\left(\xi(t)+\frac{l_{S}}{2}\right)\right. \\
\left.+\frac{1}{2}\left(\xi(t)-\frac{l_{S}}{2}\right) \operatorname{sign}\left(\xi(t)-\frac{l_{S}}{2}\right)\right], \\
D_{K}(\xi(t))=d\left[1-\frac{1}{2} \operatorname{sign}\left(\xi(t)+\frac{l_{S}}{2}\right)+\frac{1}{2} \operatorname{sign}\left(\xi(t)-\frac{l_{S}}{2}\right)\right], \\
\xi_{1}(t)=v(0, t)-w\left(l_{A}(t), t\right), \xi_{2}(t)=v\left(\left(l_{1}-l_{A}(t)\right), t\right)-w\left(l_{1}, t\right) .
\end{array}
$$

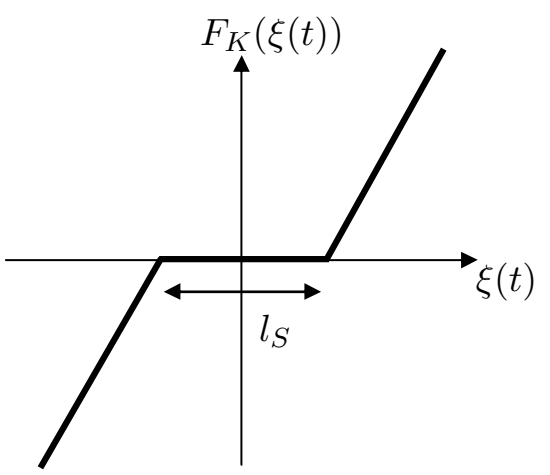

a)

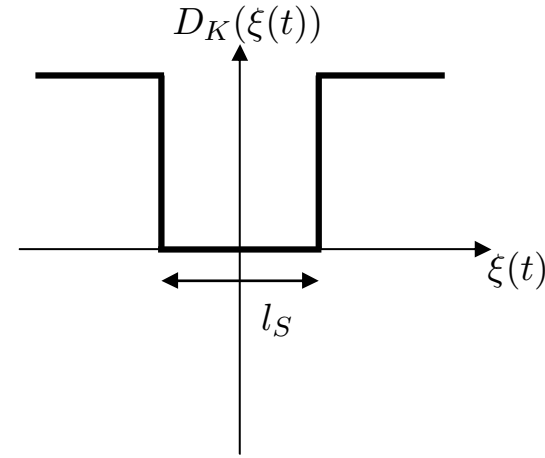

b)

Figure 4. a) Spring characteristic $F_{K}(\xi(t))$, b) Damping characteristic $D_{K}(\xi(t))$.

Fig. 4a shows the spring force $F_{K}(\xi(t))$ versus the relative position $\xi(t)$ of the contact points. The non-linear characteristic takes into account the fact that in the range of backlash no forces can be transferred. The same is valid for the assumed damping coefficient as shown in Fig. $4 b$. 


\subsection{Discretization}

The discretization of the coupled partial differential equations (5) and (6) (nonlinear and time-variant in general) together with the corresponding boundary conditions (7) and (8) is done by applying Galerkin's method (see [3], for instance). In that procedure the approximate solutions $\bar{w}\left(x_{1}, t\right)$ and $\bar{v}\left(x_{2}, t\right)$ are represented by a series expansion using selected shape functions $W_{i}\left(x_{1}\right)$ and $V_{i}\left(x_{2}\right)(i=1,2, \ldots, N)$ fulfilling all boundary conditions (7) and (8):

$$
\begin{gathered}
\bar{w}\left(x_{1}, t\right)=\sum_{i=1}^{N} u_{i}(t) W_{i}\left(x_{1}\right), \\
\bar{v}\left(x_{2}, t\right)=\sum_{i=1}^{N} u_{N+i}(t) V_{i}\left(x_{2}\right) .
\end{gathered}
$$

The coefficients $u_{i}(t)(i=1,2, \ldots, 2 N)$ have to be computed. Appropriate shape functions are modes of a corresponding problem shown in Fig. 5a and Fig. 5b.

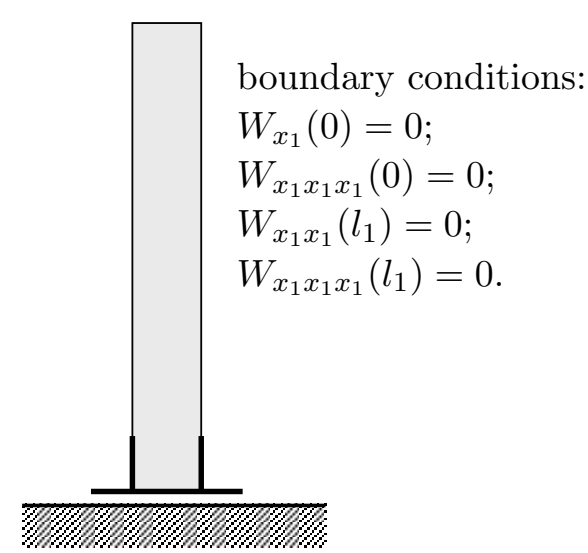

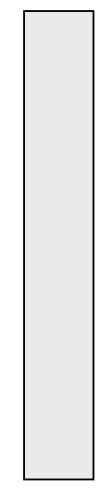

boundary conditions:

$V_{x_{2} x_{2}}(0)=0$

$V_{x_{2} x_{2} x_{2}}(0)=0$

$V_{x_{2} x_{2}}\left(l_{2}\right)=0$

$V_{x_{2} x_{2} x_{2}}\left(l_{2}\right)=0$. a)

b)

Figure 5. a) Problem "transversally movable-free", b) Problem "free-free". 
Eqs. (14) and (15) yield the final Ritz series

$$
\begin{aligned}
\bar{w}\left(x_{1}, t\right)= & \sum_{i=1}^{N} u_{i}(t)\left(\cos \left(\lambda_{i} x_{1}\right)+\frac{\cos \left(\lambda_{i} l_{1}\right)}{\cosh \left(\lambda_{i} l_{1}\right)} \cosh \left(\lambda_{i} x_{1}\right)\right), \\
\bar{v}\left(x_{2}, t\right)= & u_{N+1}(t)+u_{N+2}(t) x_{2}+\sum_{i=3}^{N} u_{N+i}(t) \\
& \times\left[\cosh \left(\kappa_{i} x_{2}\right)+\cos \left(\kappa_{i} x_{2}\right)\right. \\
& \left.-\frac{\cosh \left(\kappa_{i} l_{2}\right)-\cos \left(\kappa_{i} l_{2}\right)}{\sinh \left(\kappa_{i} l_{2}\right)-\sin \left(\kappa_{i} l_{2}\right)}\left(\sinh \left(\kappa_{i} x_{2}\right)+\sin \left(\kappa_{i} x_{2}\right)\right)\right] .
\end{aligned}
$$

The discretization applying Galerkin's procedure leads to a system of ordinary differential equations of the type

$$
\mathbf{M} \ddot{\mathbf{u}}=\mathbf{F}(\mathbf{u}, \dot{\mathbf{u}}, t) .
$$

\section{Simulation Results}

For parameter studies of an $n$-sectional telescopic system, a program based on the commercial software package MAPLE is implemented to generate the equations of motion (18) automatically starting with the system parameters.

\subsection{Case Study}

Quantitative results are presented here for a 2-sectional system. The results should illustrate the potentialities of the Galerkin model introduced in this contribution and only represent a small extract of the existing results which verify that for realistic rigid body motions of the telescopic system a low-order truncation $(N=4)$ is sufficient to get accurate results. The parameters originate from a test rig which is introduced in Chapter 4.2.

A common operating sequence of a telescopic system is simulated. The calculations are executed with $\left(l_{S}=0.01 \mathrm{~m}\right)$ and without $\left(l_{S}=0 \mathrm{~m}\right)$ clearance to show the effect of the clearance on the vibrational behavior. The system is driven by a force $F(t)$ (see Fig. 6) which acts on the rigid vehicle unit. The system starts from an initial point without any initial velocity, is accelerated by the force $F(t)$ during 4 seconds and moves with constant velocity during another 4 seconds before it is decelerated till it stops. During the simulations, the telescopic length $l_{A}(t)$ increases as shown in Fig. 7. 


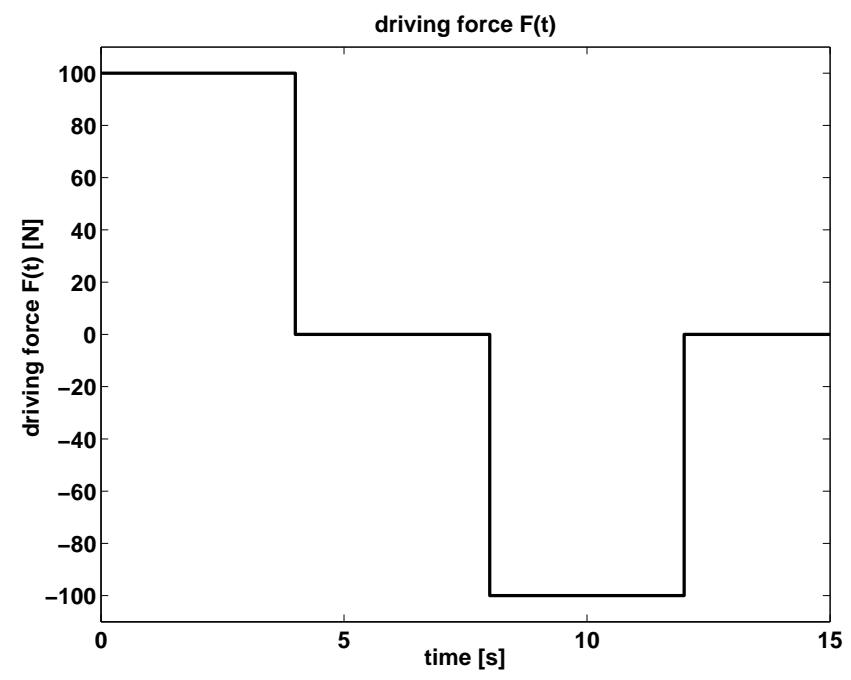

Figure 6. Driving force $F(t)$.

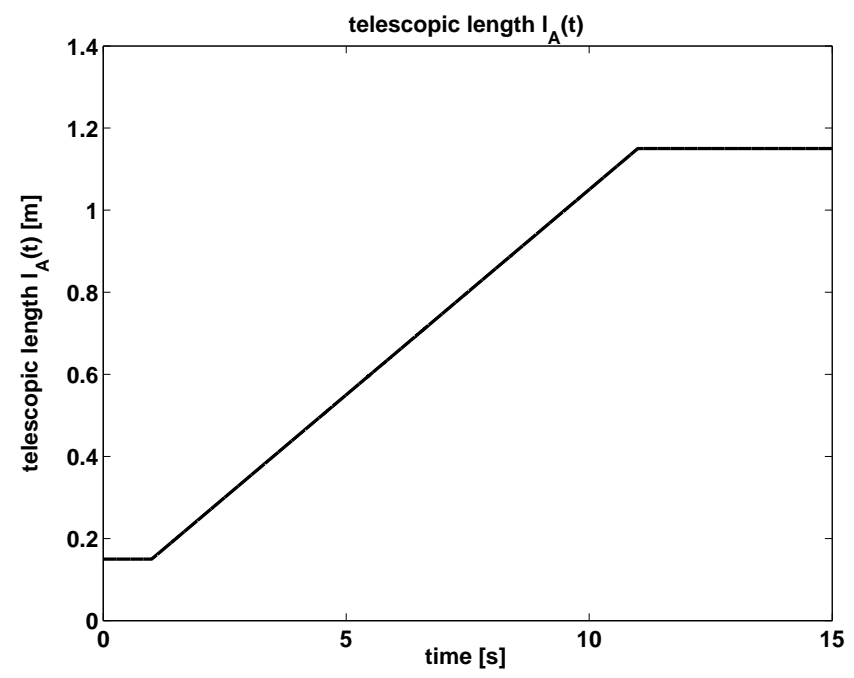

Figure 7. Telescopic length $l_{A}(t)$.

Fig. 8 shows the motion of the rigid vehicle unit, evoked by the driving force $F(t)$. Fig. 9 shows the position of the tip load relative to the bottom of the telescope. As expected, the amplitude and the oscillating period of the vibrations increase with increasing telescopic length. These vibrations have to be suppressed by an appropriate controller concept, to ensure a safe and efficient use of telescopic systems in technical applications. Moreover, the effect of the clearance on the vibrational behavior can be seen. The main cause for the distinctive results with and without clearance is the tilted position of the upper 


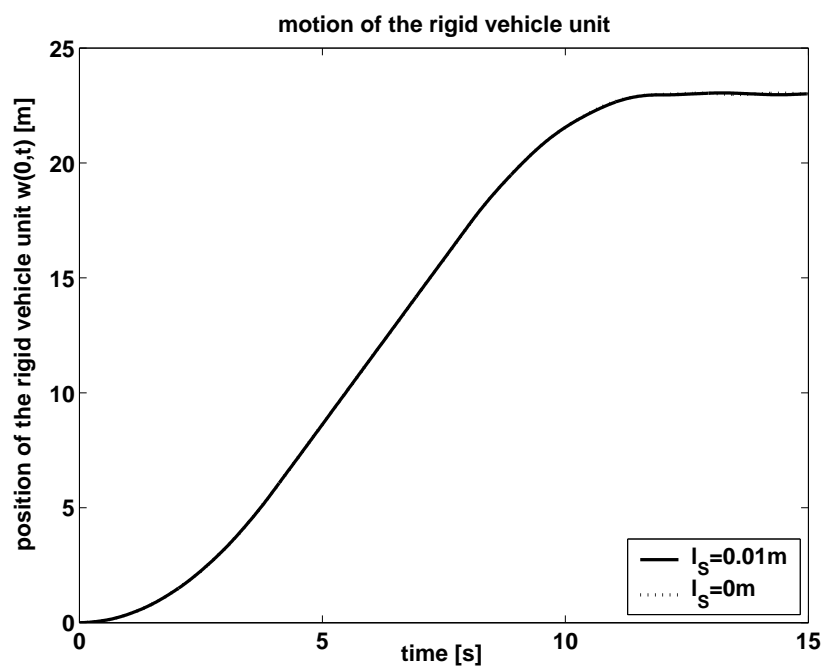

Figure 8. Motion of the rigid vehicle unit.

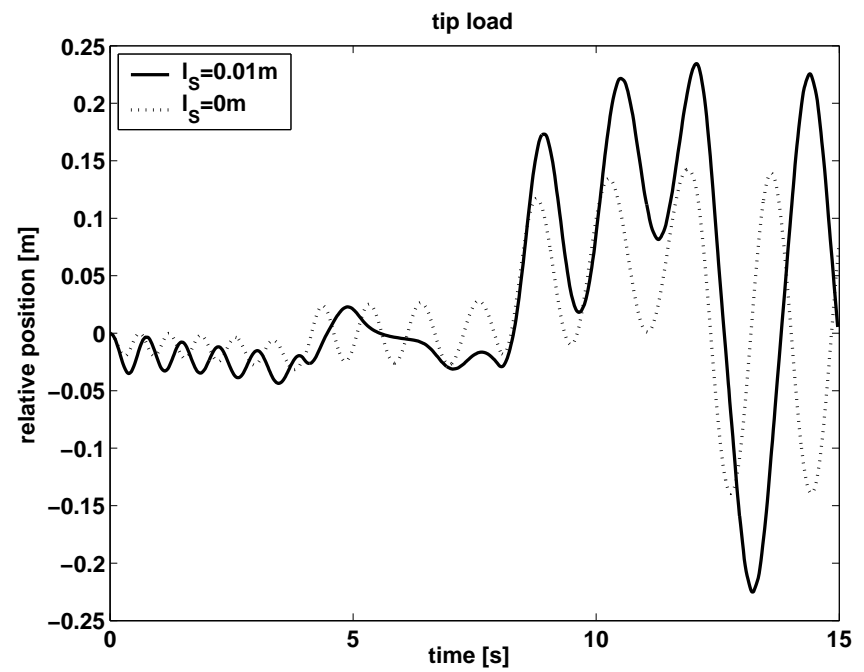

Figure 9. Position of the tip load relative to the bottom of the telescope.

segment between the lateral limitations of the guidance of the lower segment. This effect increases with increasing telescopic length and increasing clearance. The smoothness of the curve (with clearance) is due to the tip load. 


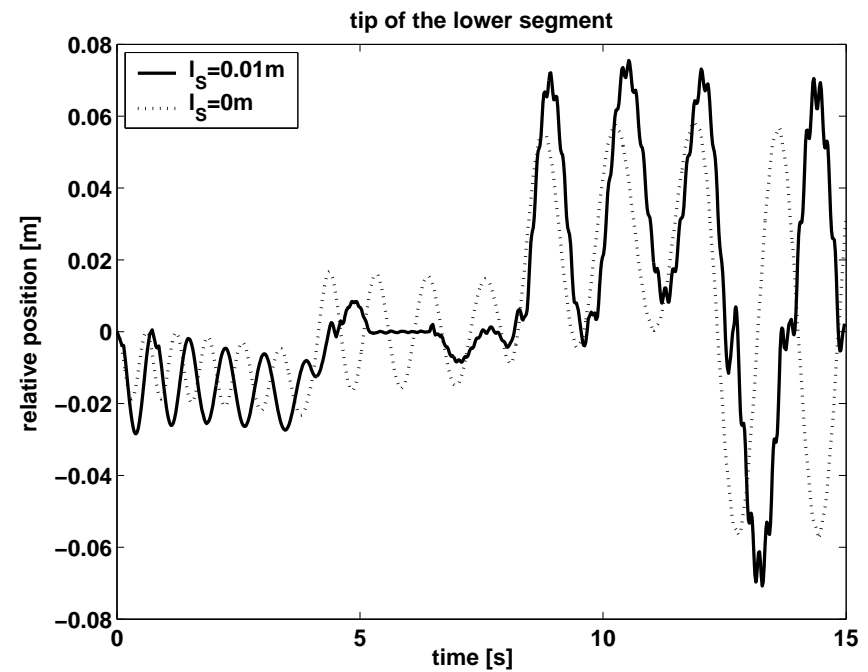

Figure 10. Position of the tip of the lower segment relative to the bottom of the telescope.

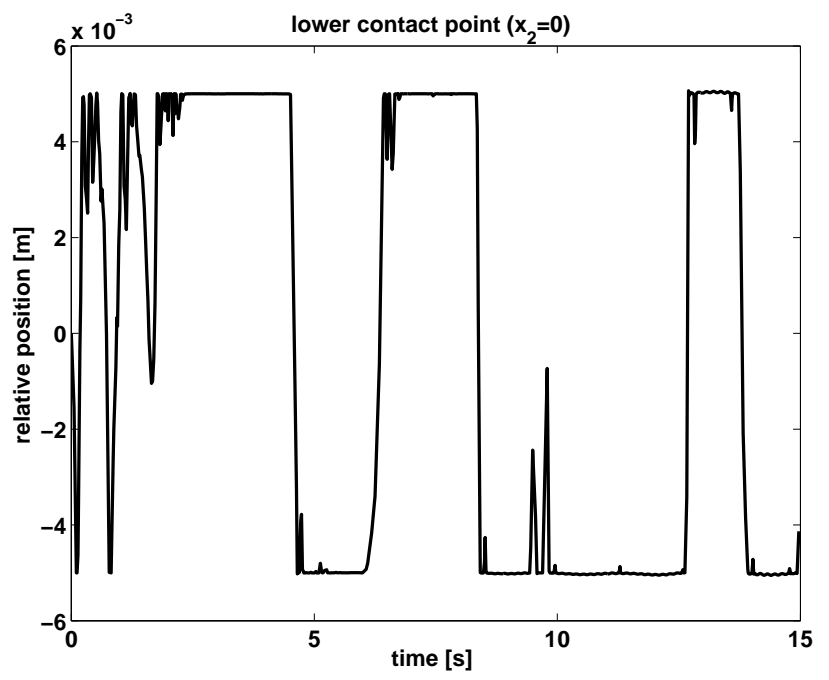

Figure 11. Position of the upper segment relative to the lower segment in the lower contact point.

Fig. 10 shows the position of the tip of the lower segment relative to the bottom of the telescope. High frequency vibrations occur in the nonlinear calculations with clearance due to the collisions of the two segments in the contact points.

Fig. 11 and Fig. 12 show the position of the upper segment relative to the lower segment in two moving contact points. During the acceleration and the deceleration the upper segment stays tilted between 
the lateral limitations of the guidance of the lower segment. The two segments stay in contact. For $F(t)=0$ the upper segment freely moves between the lateral limitations.

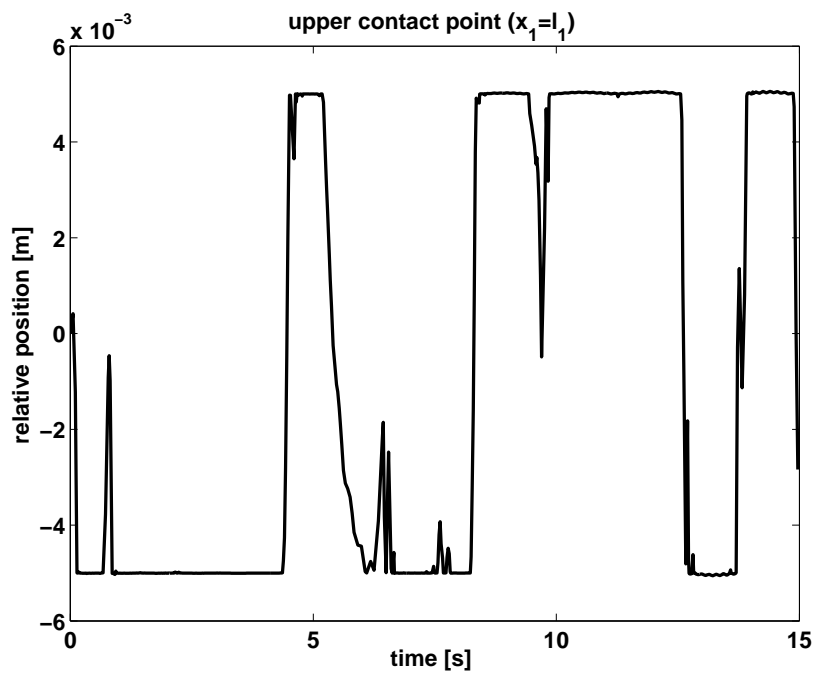

Figure 12. Position of the upper segment relative to the lower segment in the upper contact point.

\subsection{Verification of the Galerkin model}

The presented 2-sectional system was partly validated in a test rig of the Institut für Fördertechnik und Logistiksysteme, Universität Karlsruhe (TH) (see Figs. 13a, 13b and 13c).

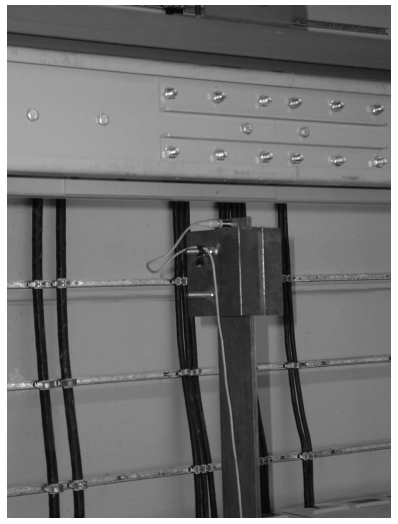

a)

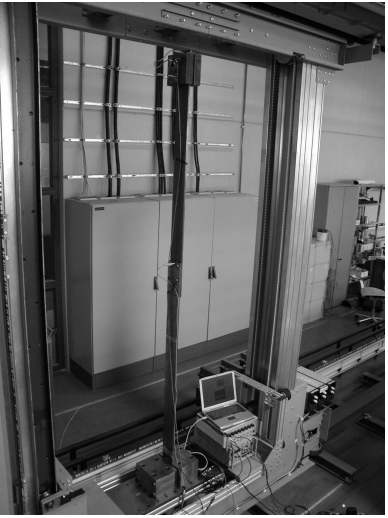

b)

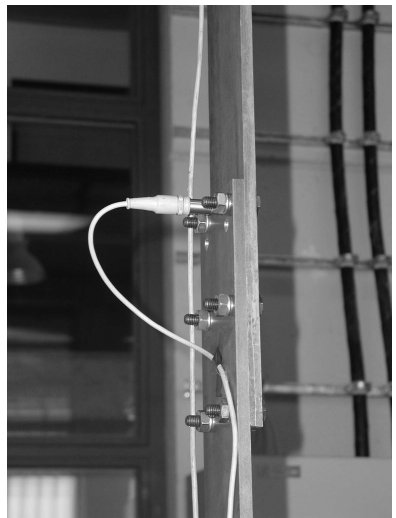

c)

Figure 13. a) Tip load $m_{L}$ b) Test rig c) Contact between beams. 
The test rig is composed of two beams of length $l_{1}=l_{2}=1.35 \mathrm{~m}$. The cross-sectional areas are $A_{1}=A_{2}=0.001 \mathrm{~m}^{2}$ and the cross-sectional moments of inertia are $I_{1}=I_{2}=0.83 \cdot 10^{-8} \mathrm{~m}^{4}$. The beam material has a density $\rho=7850 \mathrm{~kg} / \mathrm{m}^{3}$ and Young's modulus $E=2.1 \cdot 10^{11} \mathrm{~N} / \mathrm{m}^{2}$. The beams are screwed together at a fixed "telescopic" distance $l_{A}=1.15 \mathrm{~m}$ (see Fig. 13c). A real telescopic motion is not yet possible. Further parameters are the number of contact points $n=3$, mass of the load $m_{L}=17.897 \mathrm{~kg}$ (see Fig. 13a), gravity constant $g=9.81 \mathrm{~m} / \mathrm{s}^{2}$ and the contact stiffness $c=0.2375 \cdot 10^{9} \mathrm{~N} / \mathrm{m}$ to be estimated from the geometry and the material of the screws. If then the clearance between the beams is fixed to be zero $\left(l_{S}=0 \mathrm{~m}\right)$, the eigenfrequencies of the Galerkin model can be computed and compared with experimental values from the test rig (see Table I). During the experiments, the lower beam of the test rig is rigidly fixed, which is taken into consideration within the mathematical model by a very large mass $m_{T}=5000 \mathrm{~kg}$ of the vehicle. The calculation results in Table I are based on 5-term truncations (14) and (15).

Table I. Comparison of eigenfrequencies: test rig Galerkin model

\begin{tabular}{cccc}
\hline & test rig & Galerkin model & difference \\
\hline$f_{1}[\mathrm{~Hz}]$ & 0.533 & 0.53 & $0.56 \%$ \\
\hline$f_{2}[\mathrm{~Hz}]$ & 6.3 & 6.35 & $-0.79 \%$ \\
\hline$f_{3}[\mathrm{~Hz}]$ & 20.05 & 21.17 & $-5.59 \%$ \\
\hline
\end{tabular}

The good coincidence of the results in Table I shows that in the presented Galerkin model pre-stressing-effects are modeled in a satisfactory manner.

As the test rig is only partly finished at the present and clearance can not be adjusted very accurately, results from the Galerkin model are compared with results from the commercial software package ADAMS to ensure the accuracy of the presented Galerkin model. An interface to the FE-program ANSYS yields the possibility to integrate flexible bodies into the multi-body program package ADAMS. Applying this, a model of the test rig can be composed as the basis of dynamic simulations.

Figs. 14 and 15 compare the simulation results of the presented Galerkin model to those resulting from ADAMS. Drawn is the position of the tip load relative to the bottom of the telescope versus 
time. Fig. 14 shows the results of computations without clearance and Fig. 15 the results of nonlinear computations with clearance $\left(l_{S}=\right.$ $0.01 \mathrm{~m})$. The simulations are based on $m_{T}=100 \mathrm{~kg}, F=100 \mathrm{~N}$ and $N=5$. As the implementation of pre-stressing-effects and of telescopic motion is rather cumbersome in ADAMS, the computations are done without the influence of gravity and with a fixed telescopic length $l_{A}=1.15 \mathrm{~m}$.

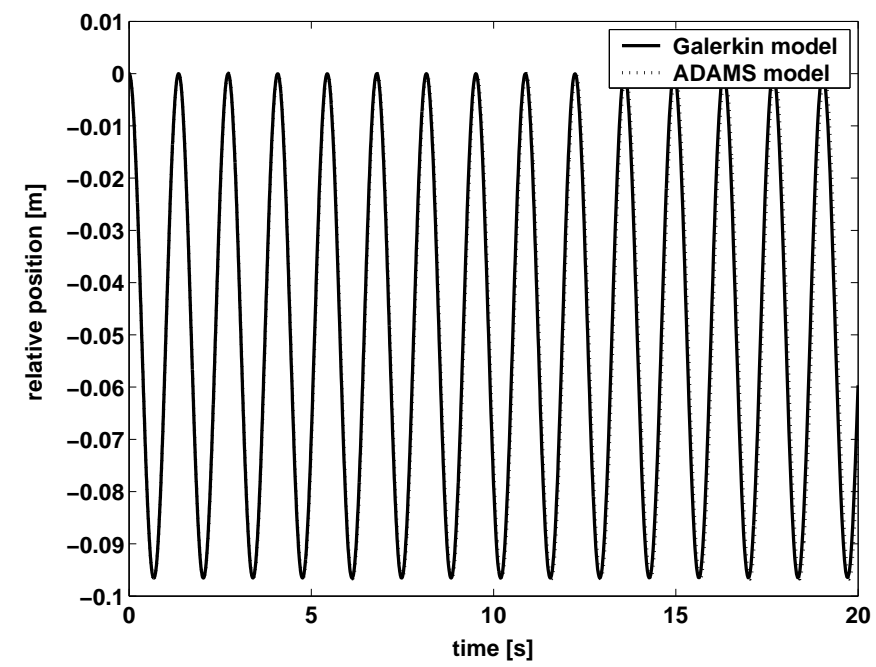

Figure 14. Linear calculations without clearance $l_{S}=0 \mathrm{~m}$.

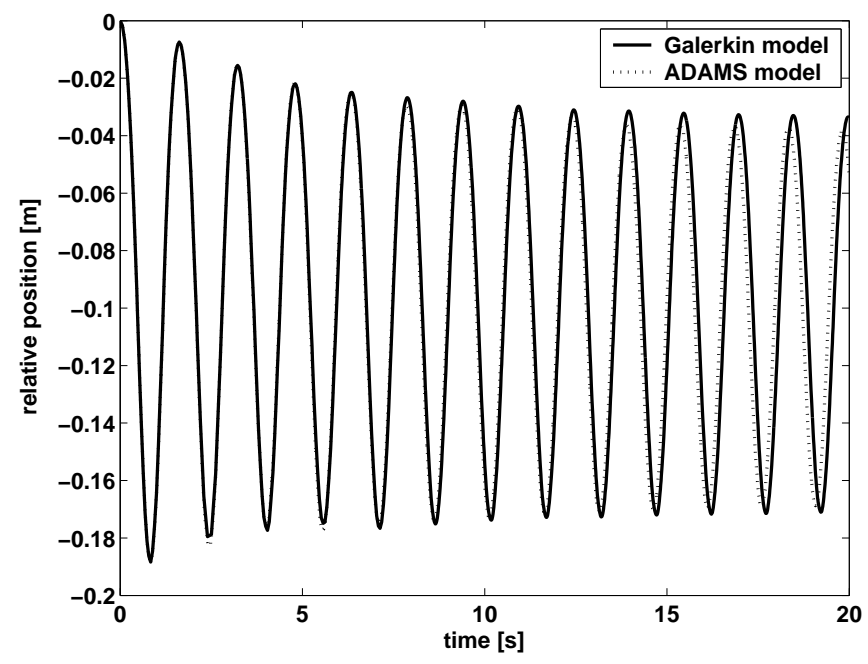

Figure 15. Nonlinear calculations with clearance $l_{S}=0.01 \mathrm{~m}$. 
Figs. 14 and 15 show that for the Galerkin model a low-order truncation is sufficient to receive good coincidence with results from the proven simulation package ADAMS. Furthermore, the simple implementation of pre-stressing-effects and of telescopic motion are important advantages of the Galerkin model. The main benefit, however, is the direct access to the system equations which allows a platformindependent treatment and the application of established control strategies for vibration suppression. In ADAMS a direct access to the system equations is not possible.

\section{Conclusions}

To improve efficiency and to overcome possible safety problems of multi-section constructions during extending and retracting motion of the sections, a vibration suppression in structural systems of variable geometry seems to be useful. To achieve this, an appropriate modeling of the system and a detailed dynamic analysis together with the development of an efficient control strategy are the essential problems to be treated. For slender beam-shaped structural members, the present contribution has suggested an approach to find a good modeling with a justifiable computational expense.

The key idea is the description of the multi-section beam system with reference to an inertial frame and the formulation of contact conditions in a form straightforward to handle. Based on this, the governing boundary value problem was derived applying Hamilton's principle, and a series expansion was introduced to generate a system of nonlinear ordinary differential equations as the corresponding truncated mathematical model. Displaying the set of equations in semi-symbolic form by MAPLE makes a platform-independent treatment possible, and the direct access to the system equations allows the application of established control strategies for vibration suppression to be the final objective.

The simulation results to examine the vibrational behavior verify that for realistic rigid body motions of the telescopic system a loworder truncation is sufficient and the computational effort is low, even for systems with clearance.

In a future paper the development of a controller concept for preventing harmful vibrations will be discussed. 


\section{Acknowledgements}

The authors thank Deutsche Forschungsgemeinschaft (DFG) for supporting this project under grant AR 160/15-1.

\section{References}

1. Fujita, K., Shiona Y., Koike K., Koseki T. Multibody Dynamics of the 2 Section Ladder Model Extending and Retracting Each Other. Proc. of ASME-DETC'03, Chicago (USA), Sept. 2-6, 2003, VIB-48367.

2. Waltersberger B., Keppler R., Wauer J. Stoßsimulation mittels einseitiger FederDämpfer Kontakte. PAMM 3, 2004, in press.

3. Riemer M., Wauer J., Wedig W. Mathematische Methoden der Technischen Mechanik. Springer, Berlin, 1993.

blacksburg.tex; 18/02/2005; 11:45; p.16 